\title{
CONFÉRENCE
}

\section{Techniques alternatives en imagerie}

\section{Dr Laurent Hennequin}

Metz

L'imagerie 3D par faisceau conique est devenue la référence incontournable en imagerie dentaire. D'autres modalités d'imagerie maxillo-faciale sont utiles, qui seront passées en revue dans cet exposé. Le scanner reste nécessaire dans l'exploration conjointe du rachis cervical et de l'encéphale (traumatismes, tumeurs), en raison de son caractère exhaustif (balayage crâniofacial large ou corps entier) et de sa meilleure résolution en densité. L'IRM apporte son excellente résolution en densité, permettant par exemple l'étude des glandes salivaires - en pathologie inflammatoire, lithiasique ou tumorale -, l'exploration des tumeurs maxillo-mandibulaires, ou l'exploration des pathologies sinusiennes (sinusite maxillaire «plus"). L'échographie, d'accès facile et bon marché, permet l'étude des adénopathies cervicales, l'analyse des parties molles cervicales et faciales, et guide les ponctions, à visée cytologique ou histologique, et les aspirations ou les drainages. L'artériographie n'a plus sa place, remplacée par l'angioscanner artériel et veineux (cartographie vasculaire avant greffon par lambeau pédiculé). Plus généralement le rôle du radiologue, au service du chirurgien maxillo-facial et ondontologiste, terminera l'exposé, en l'étendant à certaines techniques de radiologie interventionnelle, et à la transmission des images au clinicien, via les réseaux et les PACS. 